\section{IN BRIEF}

\section{$\Rightarrow$ PROSTATE CANCER}

\section{Immunogenomic features of $\mathrm{mCRPC}$ revealed}

An analysis of biopsy samples from 124 men with metastatic castration-resistant prostate cancer (mCRPC) provides data on the relevance of genomic instability to the immunological landscape. Overall, 10 patients (8.1\%) had defective mismatch repair (dMMR), which was also associated with greater $T$ cell infiltration and higher PD-L1 expression. Researchers then compiled a set of dMMR signatures, based on the presence of mutations in genes associated with DNA repair processes. Associations between immune-related gene expression and this dMMR-associated phenotype were then explored: notably, a positive association was observed between the proportion of dMMR activity and total immune infiltrate. When data from multiple biopsy sites were pooled, specific associations were observed between dMMR and 12 immune-checkpoint-related genes, including PDL1, PDL2, and TIM3. These findings have implications for the development of immune-related biomarkers for patients with mCRPC.

ORIGINAL ARTICLE Nava Rodrigues, D. et al. Immunogenomic analyses associate immunological alterations with mismatch repair defects in prostate cancer. J. Clin. Invest. https://doi.org/10.1172/JCl121924 (2018)

\section{$\Rightarrow$ LUNG CANCER}

\section{Erlotinib effective in the neoadjuvant setting}

The tyrosine kinase inhibitor (TKI) erlotinib has been shown to improve the outcomes of patients with advanced-stage non-small-cell lung cancer (NSCLC), although effectiveness in the neoadjuvant setting is less well established. In a single-arm phase II trial, 19 patients with stage IIIA-N2 EGFR-mutated NSCLC received erlotinib for 56 days before surgery. A total of 14 patients underwent surgery, of which 13 (68.4\%) underwent radical resection, which was the primary end point of the trial. The majority of patients (17/19) achieved disease control, with a median disease-free survival duration of 11.2 months and an overall survival duration of 51.6 months from the start of neoadjuvant therapy. These findings provide preliminary evidence for the use of TKIs in the neoadjuvant setting in patients with NSCLCs harbouring EGFR alterations.

ORIGINAL ARTICLE Xiong, L. et al. Erlotinib as neoadjuvant therapy in stage IIIA (N2) EGFR mutation-positive non-small cell lung cancer: a prospective, single-arm, phase II study. Oncologist https://doi.org/10.1634/theoncologist.2018-0120 (2018)

\section{IMMUNOTHERAPY}

\section{PD-1 mRNA predicts response to therapy}

Response rates to immune-checkpoint inhibitors vary dramatically between patients with different tumour types, although the underlying reasons remain largely unknown. Now, in an analysis of TCGA data, including data from $>10,000$ samples and 34 different tumour types, researchers explored the relationship between the level of PD-1 mRNA and sensitivity to ICls. A positive correlation $(r=0.91)$ was observed between the percentage of tumours with a 'high' PD-1 mRNA level (defined as the proportion of samples above the $80^{\text {th }}$ percentile for PD-1 mRNA level) and overall response rates to anti-PD-1 antibodies reported in the literature. Furthermore, high PD1 mRNA levels were strongly correlated with expression of $\mathrm{CD}^{+}$ T cell-related genes. These observations were confirmed in an independent set of 773 tumour biopsy samples. These data demonstrate the potential of PD-1 mRNA as a biomarker of response to anti-PD-1 antibodies.

ORIGINAL ARTICLE Paré, L. et al. Association between PD1 mRNA and response to anti-PD1 monotherapy across multiple cancer-types. Ann. Oncol. https://doi.org/10.1093/ annonc/mdy335 (2018)

\title{
Signatures IMPRES and might turn the TIDE in predicting responses
}

Immune-checkpoint inhibitors (ICIs) can provide major therapeutic benefits, but most patients do not respond to these agents. Now, two studies published in Nature Medicine highlight the potential of tumour transcriptome profiling to improve the allocation of ICIs.

In one study, a computational tumour immune dysfunction and exclusion (TIDE) framework was developed using data from $>33,000$ human tumour specimens by testing the effects of interactions among the candidate genes with either cytotoxic T cells or immunosuppressive cell signatures on the risk of death. Thus, "TIDE differs from existing biomarkers by providing signatures of both $\mathrm{T}$ cell dysfunction in immunologically 'hot' tumours and T cell exclusion in 'cold' tumours," explains Shirley Liu. "Instead of deriving response biomarkers from immunotherapy cohorts, we derived these signatures from publicly available treatment-naive tumour data sets with transcriptome and patient survival information," Liu adds. "Such data have been used extensively to examine the impact of a particular gene or pathway on patient survival; our approach now enables investigators to determine whether these relationships correlate with immune function," says co-author Kai Wucherpfennig.

When applied to the available pretreatment transcriptomic data from patients with melanoma who subsequently received ICIs, TIDE consistently out-performed all other candidate predictive biomarkers tested, including PD-L1 levels, tumour mutational burden, and an IFN $\gamma$ signature. Importantly, the component interactions can reveal mediators of ICI responsiveness. For example, resistance was predicted by high mRNA levels of SERPINB9, an IFN $\gamma$-regulated gene encoding a potentially targetable protease that can degrade granzyme $\mathrm{B}$.

In the second study, an immuno-predictive score, IMPRES, was developed to predict immunemediated spontaneous regression of neuroblastoma. This score is calculated on the basis of 15 rational pairwise relationships between the expression levels of inhibitory or activatory immune-checkpoint genes. As well as predicting neuroblastoma regression, high IMPRES scores were found to be associated with immunologically hot tumours and longer overall survival in patients with untreated metastatic melanoma, prompting evaluation of the value of IMPRES in predicting responses to ICIs in this setting.

"Using pretreatment or on-treatment tumour samples, we found that this simple scoring system robustly predicts the responses of patients with melanoma to different ICIs," Noam Auslander summarizes. Across 1 new and 9 published data sets comprising 297 samples in total, IMPRES performed better than other transcriptome-based predictors, identifying almost all true responders while misclassifying $<50 \%$ of nonresponders. Notably, the similarities with melanomas in regard to immune-related gene expression hint at the potential of ICIs and IMPRES in neuroblastoma.

"We hope that the clinical utility of IMPRES will be further tested and validated, and we would like to run a clinical trial," states Auslander. "Our hope is that IMPRES can ultimately be used to identify patients with cancers that are completely resistant to ICIs and thus should be spared from the associated adverse effects and costs," she concludes.

Moving forward, the developers hope to refine TIDE and IMPRES for use with other diverse cancers with distinct immunological phenotypes.

David Killock

ORIGINAL ARTICLES Jiang, P. et al. Signatures of T cell dysfunction and exclusion predict cancer immunotherapy response. Nat. Med. https:// doi.org/10.1038/s41591-018-0136-1 (2018)| Auslander, N. et al. Robust prediction of response to immune checkpoint blockade therapy in metastatic melanoma. Nat.Med. https://doi.org/10.1038/ 541591-018-0157-9 (2018) 